\title{
THERMODYNAMICS AND SOURCES OF SOLAR HEAT, 1846-1862
}

\author{
Frank A. J. L. James*
}

IN 1859 Charles Darwin in chapter nine of the Origin of Species showed how he had calculated that the age of the Weald was three hundred million years and that consequently the age of the earth was considerably greater than that. ${ }^{1}$ Darwin of course needed such a long period of time for the process of evolution by natural selection to occur. Arguments which showed that the earth could not be that old would therefore cast serious doubt on his theory. Such views were advanced in 1862 by William Thomson, later Lord Kelvin, professor of Natural Philosophy at Glasgow. ${ }^{2}$ He specifically challenged the result of Darwin's calculation of the age of the Weald by arguing that the sun could not have emitted its heat and light for that length of time. ${ }^{3}$ The consequences of this assertion for the biological and geological sciences for the remainder of the nineteenth century have already been delineated by Burchfield. ${ }^{4}$ What I wish to do in this paper is to show that the theoretical basis of Thomson's 1862 assertion had not been specifically developed as a response to Darwin, but that it was a consequence of the formulation of the first two laws of thermodynamics. ${ }^{5} \mathrm{I}$ shall also show that Thomson's work was not done in isolation but that the question of the maintenance of solar energy was a serious concern of a number of physicists who had formulated the laws of thermodynamics.

The possibility that the sun might stop emitting light and heat had been realised before the nineteenth century. Newton appears to have

* Dept. of Humanities, Sherfield Building, Imperial College, University of London, South Kensington, London, SW7 2AZ. I wish to thank Dr M. B. Hall, Dr J. D.Burchfield, and Prof. G. J. Whitrow for their comments on earlier versions of this paper. I also thank the University Library Cambridge for permitting me to study the correspondence of G. G. Stokes and William Thomson. A shorter version of this paper was presented to the History of Astronomy and Physics section of the XVIth International Conference of the History of Science in Bucharest in August 1981.

${ }^{1}$ C. Darwin, On the Origin of Species by Means of Natural Selection, London, 1859, pp. 286-7.

${ }^{2}$ William Thomson, Lord Kelvin (1824-1907). The main collection of his MSS is in the University Library Cambridge (ULC add MS 7342); there is also a smaller collection in the University of Glasgow Library. Most of Thomson's scientific papers were collected in his Mathematical and Physical Papers, 6 volumes, Cambridge, 1882-1911; volumes 1-3 were edited by Thomson and volumes 4-6 were edited by J. Larmor. These will be cited as Thomson Papers. Thomson's Popular Lectures and Addresses, 3 volumes, London, 1889-94 will be cited as Thomson Lectures.

${ }^{3}$ W. Thomson, 'On the Age of the Sun's Heat', Macmillan's Magazine, 1862, 5, 388-93; Lectures I, 356-75. The explicit reference to the Weald occurs on p. 368.

${ }_{5}^{4}$ J. D. Burchfield, Lord Kelvin and the Age of the Earth, New York, 1975.

5 For accounts of the development of the laws of thermodynamics see $\mathrm{C}$. Truesdell, The Tragicomical History of Thermodynamics 1822-1854, New York, 1980; W. L. Scott, The Conflict between Atomism and Conservation Theory 1644-1860, London, 1970; Y. Elkana, The Discovery of the Conservation of Energy, London, 1974; T. Kuhn, 'Energy Conservation as an Example of Simultaneous Discovery' in M. Claggett (ed.), Critical Problems in the History of Science, Madison, 1969, pp. 321-56.

ISSN 0007-0874/1982/July/1502-0155 \$02.00/0/C British Society for History of Science 
thought that this was the case and proposed, albeit ambiguously, that the material of the sun which had been wasted by its emission of heat and light might be restored by comets falling into it. ${ }^{6}$ Euler argued in his Letters to a German Princess that the sun could not be emitting material particles since this would lead to its rapid diminution, which was observed not to occur. ${ }^{7}$ This argument he used to justify his belief that light was undulatory. According to this theory, Euler argued, the sun was behaving as a 'bell' in sending forth its undulations into the all pervading luminiferous aether. ${ }^{8}$

When the undulatory theory gained wide acceptance in the 1820 s and ' 30 s the problem of how the sun generated its heat and light, ceased for a while to be of any great import. In 1834 John Herschel could call the problem a 'great mystery' and

If conjecture might be hazarded, we should look rather to the known possibility of an indefinite generation of heat by friction, or to its excitement by the electric discharge, than any actual combustion of ponderable fuel, whether solid or gaseous, for the origin of the solar radiation. ${ }^{9}$

Such views could quite easily be put forward when there was no need to consider the conservation of energy. But if heat and light were forms of energy, ${ }^{10}$ the problem became entirely different.

By 1840 the rate of emission of solar energy generally accepted was that which had been determined by C.-S.-M. Pouillet. ${ }^{11} \mathrm{He}$ found using a pyroheliometer that (assuming there was no terrestrial atmosphere to absorb the solar heat) in one minute the sun would raise the temperature of one gramme of water placed on the surface of the earth $1.7633^{\circ} \mathrm{C} . .^{12}$ This implied that, in one minute, one square centimetre of the solar surface emitted enough heat to raise the temperature of a gramme of water on the

${ }^{6}$ I. Newton, The Mathematical Principles of Natural Philosophy, (Motte-Cajori translation), Berkeley, 1960 , pp. 540-2. This aspect of Newton's work is discussed by D. Kubrin, 'Newton and the Cyclical Cosmos: Providence and the Mechanical Philosophy', 7. Hist. Ideas, 1967, 28, 325-46. In this passage in the Principia Newton only discussed novae. However Newton does seem to have been deliberately ambiguous as to what he thought was the source of solar heat. This is apparent from his conversation with Conduit printed in D. Brewster, The Life of Sir Isaac Newton, London, 1831, pp. 363-6.

${ }^{7}$ L. Euler, Lettres a une Princesse d'Allemagne, 2 volumes, St Petersburg, 1758, Reprinted in Leonhardi Euleri Opera Omnia, 3 series, Berlin, Göttingen, Leipzig, Heidelberg, Zürich, 191 1-, 3rd series, volumes 11 and 12 (edited by A. Spieser), Zürich, 1960. Letter 17.

${ }^{8}$ Ibid., letter 19.

${ }^{9} \mathrm{~J}$. F. W. Herschel, A Treatise on Astronomy, London, 1834, art. 337.

${ }^{10}$ See R. J. McRae, 'The Origin of the Conception of the Continuous Spectrum of Heat and Light', University of $W$ isconsin, Ph.D. thesis, 1969 and S. G. Brush, 'The Wave Theory of Heat: A Forgotton Stage in the Transition from the Caloric Theory to Thermodynamics', B.7.H.S. 1970, 5, 145-67.

1 Claude-Servais-Mathias Pouillet (1790-1868), 'Mémoire sur le chaleur solaire, sur les pouvoirs rayonnants et absorbants de l'air atmosphérique, et sur la température de l'espace', Compies Rendus, 1838, 7, 24-65. Translated into English as 'Memoir on the solar heat, on the radiating and absorbing powers of atmospheric air, and on the temperature of space', Taylor's Sci. Mem., 1846, 4, 44-90. J. F. W. Herschel had also done some work on determining the solar constant; see his Outlines of Astronomy, London, 1849, art. 397. Pouillet's figure was, as we shall see, that generally adopted since in his paper he gave his data and listed his assumptions concerning the transfer of heat through the terrestrial atmosphere. He also provided the value for the rate of emission in easily utilisable forms. For a detailed account of this work see P. A. Kidwell, 'Prelude to Solar Energy: Pouillet, Herschel, Forbes and the Solar Constant'Ann. Sci., 1981, 38, 457-76.

${ }_{12}$ Pouillet, Sci. $M e m$., Op. cit. (I1), p. 50. The accepted present day value is $2^{\circ} \mathrm{C}$. 
solar surface $84888^{\circ} \mathrm{C} .{ }^{13}$ From this figure Pouillet calculated that each year the temperature of the sun should drop $4 /(3 c)^{\circ} \mathrm{C}^{14}$ where $c$ was the specific heat of the sun, assuming perfect conductibility throughout its whole sphere.

In addition to explaining how such an enormous amount of energy could be generated, any theory of the sun also had to take into account two other observational constraints. Firstly during the period in which human records had been kept (since Hipparchos say) the sun had not been observed either to contract or expand. And, secondly, the rate at which this radiation had been emitted had to be fairly constant over this period at least.

Julius Robert Mayer, ${ }^{15}$ who in the early 1840 s had formulated a version of the principle of the conservation of energy, thought that the vast rate of emission of solar heat meant that 'in terms of human conceptions the sun is an inexhaustible source of physical energy'. ${ }^{16}$ But he wrote in 1848 that according to the principle of the conservation of energy

Every incandescent and luminous body diminishes in temperature and luminosity in the same degree as it radiates light and heat, and at last, provided it be not repaired from some other source of these agencies, becomes cold and non-luminous. ${ }^{17}$

Mayer, using Pouillet's data, ${ }^{18}$ computed the rate at which the sun was emitting radiation and arrived at the conclusion that unless there was no replenishment, the sun ought to $\mathrm{cool} 1.8^{\circ} \mathrm{C}$ annually. That is, in a period of 5000 years the sun should have cooled $9000^{\circ} \mathrm{C} .{ }^{19} \mathrm{He}$ thought that 'this amazing radiation ought, unless the loss is by some means made good, to cool considerably even a body of the magnitude of the sun'20 and that the sun would ultimately become cold.

This would have disastrous consequences for the earth since, as Mayer had noted in 1845 'the stream of this [solar] energy which . . pours over our earth is the continually expanding spring that provides the motive power for terrestrial activities'. ${ }^{21}$ The investigation of the source of the sun's

13 Ibid., p. 53.

14 Ibid., p. 54.

15 Julius Robert Mayer (1814-1878). For an account of his life and English translations of most of his papers see R. B. Lindsay, Julius Robert Mayer: Prophet of Energy, Oxford, 1973.

${ }_{6}$ J. R. Mayer, Die organische Bewegung in ihiem Zusammenhang mil dem Stoffwechsel. Ein Beitrag zur Naturkunde, Heilbronn, 1845. Translated into English as 'The Motions of Organisms and their Relation to Metabolism. An Essay in Natural Science' in Lindsay, Op. cit. (15), pp. 75-145, p. 99.

${ }^{17}$ J. R. Mayer, Beiträge zur Dynamik des Himmels, in populärer Darstallung, Heilbronn, 1848. Translated into English by H. Dubus as 'On Celestial Mechanics', Phil. Mag., 1863, IV, 25, 241-8, 387-409, 417-28; p. 241 .

18 Ibid., 244-5.

19 Ibid., 245. In performing this calculation Mayer assumed that the material of the sun had the same specific heat as water and that the sun emitted its heat uniformly from its whole mass. In his paper he did not specify the solar radius which he used, but from my calculation he appears to have assumed the radius to be 712200 kilometres. This agrees (approximately) with his statement (Ibid., 246) that the sun's diameter is nearly 112 times larger than the earth's.

20 Ibid., 245.

${ }^{21}$ Mayer, 'The Motions..., in Lindsay, Op. cit. (15), p. 99. 
energy was therefore not only a very interesting scientific problem, but also one which was perceived by a founder of thermodynamics as being fundamental to the very existence of man himself. Mayer turned his attention to the problem in a paper which he sent to the Académie des Sciences in Paris in 1846, but only an extract from this dealing with the mechanical equivalent of heat was published;22 two years later he published at his own expense, the whole paper as Beiträge zur Dynamik des Himmels.

Mayer examined the various mechanisms for the production of solar heat that had been advanced in the past. He specifically rejected the bell analogy by asking what 'undiminished force' kept the sun behaving as a bell. ${ }^{23}$ Further he showed that even on the most favourable supplementary hypotheses the heat of the sun could not have been sustained for even 5000 years by chemical processes, ${ }^{24}$ and that the sun could not be a heated body steadily losing heat. ${ }^{25}$ Possible new causes of solar heat which Mayer postulated included the rapid rotation of the sun on its axis; this he also rejected since he could not think of any frictional agent against which the sun's surface could resist.

Energy considerations provided a test of whether any particular mechanism could provide sufficient solar heat within the necessary observational constraints. But these constraints could not provide a solution to the problem of how the sun's energy could be generated and sustained; this had to be done by finding another source of energy by which the sun could be fueled and then checking to see if the new theory conformed to the observational constraints.

The proposition which Mayer advanced to acount for the production of solar heat was that

The wonderful and permanent evolution of light and heat be caused by the uninterrupted fall of cosmical matter into the sun. ${ }^{26}$

How Mayer devised this hypothesis is not clear. In an earlier paper he had calculated the energy of bodies falling from an infinite distance to the surface of the earth. ${ }^{27}$ Using his estimate of the mechanical equivalent of heat, Mayer found that the impact of such bodies would produce much heat. The possibility that meteoric impacts might be the source of solar heat may thus have occurred to him simply from this consideration or by his applying the equivalence of heat and mechanical energy to Newton's

\footnotetext{
22 The part of the paper which was published was entitled 'Sur la transformation de la force vive en chaleur, et réciproquement', Comptes Rendus, 1848, 27, 385-7. This makes clear, on p. 385, that the title of Mayer's paper, presented on 27 July 1846, was 'Sur le production de lumière et de la chaleur du soleil'.

${ }^{23}$ Mayer, Phil. Mag., Op. cit. (17), pp. 241-2.

24 Ibid., $245 . \quad 25$ Ibid., $246 . \quad 26$ Ibid., 387.

27 Mayer, 'The Motions . . ', in Lindsay, Op. cit. (15), p. 88.
} 
suggestion that comets falling into a star might refuel what had otherwise been a dying star. ${ }^{28}$

At no stage did Mayer consider the possibility that the meteorites fell in straight lines from space to the sun's surface, since, in his view, even though the sun's attraction acted throughout space, there was also a resisting medium (which he identified with the luminiferous aether) ${ }^{29}$ forcing bodies attracted to the sun to follow a spiral orbit. ${ }^{30}$ Therefore, since these bodies must be approaching the sun radially at a very small velocity compared to their actual velocity, it followed that there must be a conglomeration of small bodies near the sun, the existence of which was evinced by the zodiacal light. ${ }^{31}$

According to Mayer, because smaller bodies fall towards the sun faster than larger bodies, the planets would remain in the same position, their spiralling movements being imperceptible, while meteors would be attracted quickly towards the sun.$^{32}$ Now the smallest velocity at which a body could hit the sun's surface would be $1 / \sqrt{2}$ of the sun's escape velocity, while the greatest would be the escape velocity itself. After performing the calculations to determine the amount of energy released by the impact of a meteorite on the sun, Mayer concluded that

An asteroid, therefore, by its fall into the sun developes from 4600 to 9200 as much heat as would be generated by the combustion of an equal mass of coal. ${ }^{33}$

Mayer therefore concluded that meteors did not descend to the sun's surface to help in the chemical generation of solar energy, since the very act of augmentation released more energy than any chemical reaction which he could possibly imagine. ${ }^{34}$

Mayer assumed that material was still, at the present time, coming from outside the solar system to replenish the zodiacal light; this, for Mayer, was evinced by the meteors seen in the earth's atmosphere. A much greater number of meteors must consequently be by-passing the earth on their way to the zodiacal light, since the earth occupied, at any one time, only a small fraction of the space through which the meteors had to pass. Mayer realised that there would be two consequences of what was effectively the meteoric augmentation of the sun from outside the solar system. Firstly there would be an increase in the volume of the sun, which he calculated would amount to an increase in apparent solar diameter of at most one second of arc in 33000 years, i.e. an unobservable quantity over

${ }^{28}$ Mayer, Phil. Mag., Op. cit. (17), p. 397.

${ }^{29}$ Ibid., 388-9. $\quad{ }^{30}$ Ibid., $387 . \quad{ }^{31}$ Ibid., 389.

32 Ibid. Mayer did not have the problem of dealing with the asteroids since by 1848 only eight had been discovered. These being comparatively large bodies they would not fall towards the sun particularly quickly.

${ }^{33}$ Ibid., $392 . \quad{ }^{34}$ Ibid., 395. 
the historic period. ${ }^{35}$ The second, more serious, consequence was that such meteoric augmentation would increase the mass of the sun, and thus shorten the siderial year from between three eighths to three quarters of a second annually, ${ }^{36}$ which would be observable. Mayer pointed out that although, according to the undulatory theory, light did not have mass, yet

An undulating motion proceeding from a point or plane and excited in an unlimited medium, cannot be imagined apart from another simultaneous motion, a translation of the particles [of aether] themselves; it therefore follows, not only from the emission, but also from the undulatory theory, that radiation continually diminishes the mass of the sun. ${ }^{37}$

He suggested that matter from the sun forms the luminiferous medium which then spreads out through space. This emission of aether from the sun is, in terms of mass, exactly balanced by the input of meteoric matter:

The radiation of the sun is a centrifugal action equivalent to a centripetal motion. ${ }^{38}$

which

harmonizes with the supposition that the vis viva of the universe is a constant quantity. ${ }^{39}$

There was no possibility of determining when the sun would cease to emit energy since this depended on the cessation of the meteors about which Mayer possessed no information. He was not interested in cosmogonical problems; he was more concerned with what was happening now, for which evidence could be adduced, than in considering the origin of the solar system or how it had evolved:

we shall leave, however, all suppositions concerning subjects so distant from us both in time and space, and confine our attention exclusively to what may be learnt from the observation of the existing state of things. ${ }^{40}$

This emphasises the point that to Mayer the meteoric maintenance of solar heat was not an hypothetical abstraction, but a theory based on physical reasoning supported by observational evidence, such as the zodiacal light. He further suggested that other solar phenomena such as sun spots and faculae could be accounted for by supposing that they were caused by the 'most powerful meteoric processes' ${ }^{\text {'1 }}$ (i.e. large meteorites landing on the sun) creating disturbances in the solar atmosphere.

35 Ibid., $399 . \quad 36$ Ibid.

${ }^{37} \mathrm{Ibid}$., 397. In a footnote to this passage Mayer suggested that this was the reason why comet's tails pointed away from the sun.

38 Ibid. $\quad 39$ Ibid., 400.

${ }^{40}$ Ibid., $388 . \quad{ }^{41}$ Ibid., 402. 
Mayer had provided a solution to the problem of the maintenance of solar heat, which conformed with the observational constraints required of any solar theory. He admitted that the theory had problems: for example it appeared that the meteors did not approach the sun from all directions but only in the plane $30^{\circ}$ on either side of the solar equator because this was the sun spot belt, where the meteoric action could be observed. This meant that it was difficult to explain how the sun emitted heat and light uniformly over its whole surface. ${ }^{42}$ Mayer never returned to deal with this problem or any other connected with solar heat; after 1848 his personal tragedy caused him to become temporarily insane. He ceased performing any original scientific work, and indeed this 1848 paper was his last major contribution to science.

It was not until William Thomson ennunciated his version of what would later be called the second law of thermodynamics ${ }^{43}$ that he, apparently unaware of Mayer's work on the production of solar heat, turned his attention to the problem. His version of the second law stated that

It is impossible, by means of inaminate material agency, to derive mechanical effect from any portion of matter by cooling it below the temperature of the coldest of the surrounding objects. ${ }^{44}$

Thomson appears to have immediately recognised that this law meant that the sun had only a limited supply of energy. In his unpublished March 1851 theological note intended for his paper, the Dynamical Theory of Heat ${ }^{45}$ he wrote

I believe the tendency in the material world is for motion to become diffused, and that as a whole the reverse of concentration is gradually going on. I believe that no physical action can ever restore the heat emitted from the sun, and that this source is not inexhaustible. ${ }^{46}$

At this time Thomson thought that the sun was a heated body which was each year emitting a portion of its heat. ${ }^{47} \mathrm{It}$ immediately followed from the second law that the sun must eventually cease to emit any energy.

Also as a consequence of the second law the amount of energy available to man was limited:

No destruction of energy can take place in the material world without an act of power possessed only by the supreme ruler, yet transformations take place 42 Ibid.

${ }^{43} \mathrm{~W}$. Thomson, 'On the dynamical theory of heat with numerical results deduced from Mr. Joule's equivalence of a thermal unit, and M. Regnault's observations on steam', Trans. Roy. Soc. Edinb., 1851, 20, 261-98, 475-82; 1854, 21, 123-71; Papers I, 174-291. For a discussion of this paper see C. W. Smith, 'Natural Philosophy and Thermodynamics: William Thomson and the "Dynamical Theory of Heat"', B.J.H.S., 1976, 9, 293-319.

${ }^{44}$ Thomson, Op. cit. (43), art. 12. Thomson's emphasis.

${ }^{45}$ ULC add MS 7342, PA 128. This is printed in D. B. Wilson, 'Kelvin's Scientific Realism: The Theological Context', Phil. J., 1974, 11, 41-60, pp. 58-9.

${ }^{46}$ Ibid., 58.

${ }^{47}$ See W. Thomson to G. G. Stokes, 26 April 1854, ULC add MS 7656, K 69. 
which remove irrecoverably from the control of man sources of power which, if the opportunity of turning them to his own account had been made use of, might have been rendered available.

Everything in the material world is progressive. The material world could not come back to any previous state without a violation of the laws which have been manifested to man; that is without a creative act or an act possessing similar power. ${ }^{48}$

and later he wrote " "The earth shall wax old etc." The permanence of the present forms and circumstances of the physical world is limited'. ${ }^{49}$ This implies that Thomson thought that the world must have a beginning and an end. God had ensured by the physical laws that He had instilled in the universe that the existence of man was to be of limited duration. Quite how this would work in practice Thomson had not yet decided. It is far from clear whether he had explicitly linked these ideas other than at a theological level.

By early the following year (1852) Thomson had realised that the sun ultimately supplied all terrestrial energy. Writing to George Gabriel Stokes, Lucasian Professor of Mathematics at Cambridge, ${ }^{50}$ he commented that

I think that, with the exception of what might be got from tide mills, or the combustion of meteoric stones or other native metals, all [vis viva] is derived from the sun, and is merely part of the mechanical nature of the undulations which he has sent us from the epoch of the creation of the planets. ${ }^{51}$

In other words apart from the energy which could be obtained from the tides (caused mainly by the moon) and from the burning of non-organic materials, Thomson realised that the sun was almost the sole provider of energy for the earth. This sentiment which he had in common with Mayer, started him on a path which was to lead to a life long interest in solar theories. ${ }^{52}$

Thomson was not, at this time, aware of any quantitative work on solar radiation: in the same letter he had asked Stokes if there was any experimental data available to determine the amount of heat emitted by the sun. Unfortunately we do not have Stokes' reply; however two weeks later in February 1852, Thomson read a paper to the Royal Society of Edinburgh in which he referred to Pouillet's estimate of solar heat. ${ }^{53}$ The

${ }^{48}$ Wilson, Op. cit. (45), p. 58.

${ }^{49}$ Ibid., 59. The quotation is from Isaiah 51.6 .

${ }^{50}$ G. G. Stokes (1819-1903). The main collection of Stokes's MSS is kept in the University Library Cambridge (LLC add MS 7656). The correspondence between Stokes and Thomson will shortly be published in D. B. Wilson, The Correspondence between Sir George Gabriel Stokes and Sir William Thomson, Baron Kelvin of Largs, Cambridge, forthcoming.

${ }^{51}$ Thomson to Stokes, 13 January 1852, ULC add MS 7656, K 53.

52 Indeed almost the last problem which Thomson worked on was the theory of the sun, 'The Problem of a Spherical Gascous Nebula', Phil. Mag., 1908, VI, 15, 687-711; 16, 1-23; Papers V, 25483.

${ }^{53} \mathrm{~W}$. Thomson, 'On the mechanical action of radiant heat or light: on the power of animated creatures over matter: on the sources available to man for the production of mechanical effect', Proc. Roy. Soc. Edinb., 1852, 3, 108-13; Papers I, 505-10. He mentions Pouillet's work on p. 505, of which Stokes had, presumably, informed him. 
main conclusion which Thomson drew in this paper was the same as in his letter to Stokes:

Heat radiated from the sun ... is the principal source of mechanical effect available to $m^{2}{ }^{54}$

It implicitly followed from Thomson's earlier theological thoughts on the future of the sun that the period that man could exist on the earth was limited. This point he made quite explicitly in a paper which he read also to the Royal Society of Edinburgh a couple of months later entitled On a Universal Tendency in Nature to the Dissipation of Mechanical Energy. ${ }^{55}$ In this Thomson effectively defined the problem: he discussed particular consequences of his version of the second law and showed that whenever and by whatever process energy was dissipated from a source in a closed system, total restoration of that energy to that source was impossible, i.e. in every mechanical act which liberates heat it is impossible to derive fully from that heat the original quantity of mechanical effect. From this he deduced that:

within a finite period of time past the earth must have been, and within a finite period of time to come the earth must again be, unfit for the habitation of man as at present constituted, unless operations have been, or are to be performed, which are impossible under the laws to which the known operations going on at present in the material world are subject. ${ }^{56}$

Though Thomson's statements regarding the dissipation of heat apply to the universe as a whole, there can be no doubt, bearing in mind this passage and remembering that he regarded the sun as virtually the sole supplier of energy for the earth, that he was specifically considering the sun. The sun, being a finite body, was losing energy at a rate which he knew to be immense. Further, according to this view, the sun in the past had emitted more energy than it was doing now, and in the future it would emit less; both states being inimical to the existence of life on earth.

In a letter to Stokes a couple of years later Thomson indicated that at this time he had abandoned his original theory of solar heat since he said that he 'had always inclined to the primitive heat theory till rather more than two years ago'. ${ }^{57}$ Thomson's rejection of the hypothesis that the sun was a storehouse of energy which was gradually being dissipated implies that he had some notion of how long the sun should have been emitting its energy, which would be even less than Archbishop Ussher's six thousand years. ${ }^{58}$ Thomson displayed no concern with the age of the sun since he did not have an hypothesis on which he could base any estimates of its possible

\footnotetext{
54 Ibid., 510.

$55 \mathrm{~W}$. Thomson, 'On a universal tendency in nature to the dissipation of mechanical energy', Proc Roy. Soc. Edinb., 1852, 3, 139 42; Papers I, 511 -14.

56 Ibid., 514.

57 Thomson to Stokes, 26 April 1854, LLC add MS 7656, K 69.

$58 \mathrm{~W}$. Thomson, 'On the mechanical energies of the solar system', Trans. Roy. Soc. Edinb., 1854, 21, 63-80; Papers II, 1-25. p. 4 where Thomson makes this point.
} 
duration. His rejection of the primitive heat theory was simply founded on the human historical record. This qualitative approach, derived from the second law, conformed well with his theological views on the past and future of man. But he was left with the problem of establishing how this had happened in the past and what would happen in the future.

It was not long before two alternative hypotheses were proposed. At the 1853 meeting of the British Association in Hull, at which Thomson was not present, ${ }^{59}$ John James Waterston ${ }^{60}$ read a paper entitled On Dynamical Sequences in $K_{o s m o s}{ }^{61}$ in which he proposed two solutions to the problem of solar radiation. This paper, though not printed in the Report of the British Association, was fully reported in the Athenaeum and this is presumably where Thomson read it.

In this paper Waterston first argued, without going through the preliminaries of showing that previous solar theories must be false, that if enough meteorites fell onto the surface of the sun, converting their kinetic energy into heat, this would account for the enormous quantities of heat which Pouillet had shown to be emitted by the sun. Waterston showed, presumably in order to conform to the observational constraints imposed on any solar theory, that, assuming the meteors had reached their maximum velocity, i.e. the sun's escape velocity, the consequent annual expansion of the radius of the sun due to these meteoric impacts would be about 14.6 feet. ${ }^{62}$ In other words this would not be an observable increase over the historic period of astronomical observations-say 2100 years. Waterston summed up the fundamental idea of this hypothesis by saying that

[since] gravitation ... generates heat centripetally, radiation may be viewed as the escape of vis viva centrifugually ${ }^{63}$

That is, meteors approach the sun, and radiation is emitted from the sun in perpendicular directions from all over the sun's surface. The meteors in order to reach their maximum velocity had to approach the sun perpendicularly to its surface, and therefore from outside the solar system: which for Waterston was evinced by the great number of meteors observed in the earth's atmosphere, indicating that a far larger number of meteorites were falling onto the sun. Waterston had not realised that the increase of mass of the sun would have an observable effect on the motion of the earth.

${ }^{59}$ Thomson to Stokes, 20 February 1854, LLC add MS 7656, K 62 and Thomson to H. Helmholtz, 24 July 1855 (in S. P. Thompson, The Life of W'illiam Thomson, Baron Kelvin of Largs, 2 volumes, London, 1910, I, 309), make it quite clear that Thomson was not at the Hull meeting.

${ }^{60}$ John James Waterston (1811 1883). J. S. Haldane, The Collected Scientific Papers of J. J. Waterston, Edinburgh, 1928, contains a biographical sketch by Haldane of Waterston.

${ }^{61} \mathrm{~J}$. J. W'aterston, 'On dynamical sequences in Kosmos', Athenaeum, 1853, 1099-1100. This was not published in Waterston's collected papers.

62 Waterston made an elementary error in his calculation since he used an escape velocity from the sun of 545 miles per second which is a factor $\sqrt{2}$ too large. Therefore his calculation concerning the amount of meteoric matter needed was half the amount required according to his hypothesis. He also assumed that the meteoric material had the specific heat of iron and the density of water.

${ }^{63}$ Waterston, Op. cit. (61), 1099. 
The second hypothesis which Waterston proposed was that the sun as it collapsed under its own gravity, produced heat by condensation: 'In bodies that surround us we remark that cooling and contraction are generally simultaneous'. ${ }^{64} \mathrm{He}$ said that if the sun as a whole cooled uniformly $33^{\circ} \mathrm{F}$ every year ${ }^{65}$ then its radius would have to contract by 430 feet to produce this quantity of heat. ${ }^{66}$ As with his first hypothesis this amount of change in solar radius would also have been difficult to detect over the period in which astronomical observations had been made.

The idea common to both these hypotheses was that the ultimate cause of solar heat was to be found in the gravitational attraction of the sun, in 'perfect conformity' with the nebular hypothesis of Laplace ${ }^{67}$ Waterston no doubt accepted this and may have thought that following the formation of the sun and planets there must have been some material left over in the form of meteors throughout the whole solar system; evidence for this would come from the observation that some meteors coming to the earth appeared to originate from beyond its orbit. According to his second hypothesis I would suggest that Waterston may have thought that the sun was continuing the process of contraction under gravity initiated by the original nebula.

Waterston had shown that there existed answers to the question, which following the foundation of the principles of the conservation and dissipation of energy, was increasingly exercising the attention of mid nineteenth century physicists: what was the cause of the sun's heat? What Waterston had done was to show that it was quite possible to envisage physical hypotheses which could account for the production of solar heat and yet conform to the laws of thermodynamics and the observational constraints incumbent upon any solar theory. This he had done without going into the finer details of either of the hypotheses which he proposed. He appears to have been content simply to make this demonstration since he did not suggest any method to determine which of his hypotheses might be correct.

Thomson gave high praise to Waterston's suggestion that the sun's energy originated from meteoric impacts on its surface. ${ }^{68} \mathrm{He}$ commented in a paper that he read to the Royal Society of Edinburgh in April 1854 that the theory

may have occurred at any time to ingenious minds, and may have occurred

${ }^{64}$ Ibid., 1100.

${ }^{65}$ It is not clear how Waterston derived this figure.

${ }^{66} \mathrm{He}$ appears to have done this by simply considering the amount of heat produced by a body falling this distance.

${ }^{67}$ Waterston, Op. cit. (61), 1099. The main source for the nebular hypothesisis the final book of P. S. Laplace, Exposition du Systeme du Monde, Paris, 1796; it went through several changes (see S. L. Jaki, 'The Five Forms of Laplace's Cosmogony', Am. J. Phys., 1976, 44, 4-11) before the final version was published. The hypothesis became very well known and received a good deal of attention in the nineteenth century.

${ }^{6 B}$ There is no evidence to indicate whether or not Thomson attempted to contact Waterston. 
and been set aside as not worth considering; but it was never brought forward in any definite form, so far as I am aware, until $\mathrm{Mr}$ WATERSTON communicated to the British Association, during its last meeting at Hull, a remarkable speculation on cosmical dynamics, in which he proposed the Theory that solar heat is produced by the impact of meteors falling from extra-planetary space, and striking his surface with velocities which they have acquired by his attraction. ${ }^{69}$

This was the highest praise Thomson gave Waterston's work; the paper in which he proposed his version of the meteoric theory, was, as we shall see, written with caution. Indeed in it Thomson noted many fallacies which occurred in Waterston's work, proceeding to construct his own theory to take account of them. A possible reason for this caution was that Thomson's ex-supervisor at Cambridge, and president of the Association for that year, William Hopkins, commented that while Waterston's paper suggested 'important hints and valuable lines of enquiry', ${ }^{70}$ caution should be exercised in regarding his work as representing 'determined scientific truth'? ${ }^{71}$

Thomson took heed of Hopkins's warning. Using a form of the reductio ad absurdum argument, he discussed three possible theories of solar heat, and showed that two of them could not supply sufficient energy for the sun, implying that the third did. Besides Waterston's meteoric hypothesis the other two theories were first, that the sun was a hot body losing heat, and second that chemical reactions within the sun were causing the heat. ${ }^{2} \mathrm{He}$ maintained that the former proposition was untenable since it could not provide the sun with six thousand years of energy ${ }^{73}$ The second proposition he showed to be false because of the enormous amount of matter required, assuming similar chemical reactions to those observed on earth, for the sun to emit heat for any significant period of time. ${ }^{74}$

Until the laws of thermodynamics were established, there had been no reason to suppose that the sun was running down and such theories as those which Thomson refuted could be easily advanced. The second law also precluded what Thomson called "anti-radiation"75 (i.e. heat coming to the sun from some other source) from restoring energy to the sun, for he argued, there was no other body in the solar system at a higher temperature than the sun, and therefore no heat could pass to the sun from anywhere else in the solar system. Consequently it became necessary to devise a new mechanism by which the sun could be supplied with energy.

It was this necessity which enabled Thomson to adopt Waterston's meteoric hypothesis with apparent ease, since it did, after all, provide an explanation for the production of solar heat. Thomson thought that some

69 Thomson, Papers, Op. cit. (58), p. 4.

70 Athenaeum, $1853,1100 . \quad 71$ Ibid.

72 Thomson, Papers, Op. cit. (58), p. 3.

73 Ibid., $4 .{ }^{74}$ Ibid., $10 .{ }^{75}$ Ibid., 3. 
heat must be generated by meteors falling onto the sun's surface, since Joule had shown that they generated heat in passing through the earth's atmosphere. ${ }^{76}$ Thomson therefore argued from this that the meteoric hypothesis

is in fact not only proved to exist as a cause of solar heat, but it is the only one of all conceivable causes which we know to exist from independent evidence. ${ }^{77}$

This did not prove that meteors were the sole cause, but since he had shown that the other 'conceivable causes' were entirely insufficient to supply the required amount of solar heat, it followed that the meteoric hypothesis must be sufficient. Indeed writing to Stokes in March 1854, Thomson expressed himself in a manner which leaves no doubt that he firmly believed in the truth of the hypothesis:

There must be a great deal of ... [iron] about the sun, seeing we have so many iron meteors falling in [the earth's atmosphere], and there must be immensely more such falling in to the sun. I find the heat of combustion of a mass of iron $\mathrm{w}$ [oul]d be only $1 / 34000$ of the heat derived from potential energy of gravitation, in approaching the sun. Yet it w[oul]d take 2000 pounds of meteors per sq. foot of the sun, falling annually to account for his heat by gravitation alone. ${ }^{78}$

At this time Thomson evidently accepted Waterston's original version of the theory in which the meteors came from outside the earth's orbit, since 2000 pounds of meteors was the mass required only if the meteors had achieved their maximum velocity when they reached the sun's surface. $\mathrm{He}$ argued that the chemical energy produced by the burning of meteoric material was insignificant compared with the heat produced by the conversion of the meteorite's energy due to motion into heat and light which it generated on impact. ${ }^{79}$

Stokes's response to Thomson's theory, after some prodding by Thomson, ${ }^{80}$ was to say that he knew no objection against it. Stokes also ${ }^{81}$ rejected William Herschel's suggestion made in $1795^{82}$ that the sun possessed an atmosphere which alone produced the solar light and heat, adding that he had always been 'in the habit' of assuming that the sun was

${ }^{76}$ J. P. Joule, 'On Shooting Stars', Phil. Mag., 1848, III, 32, 349-51; in The Scientific Papers of James Prescott Joule, 2 volumes, London, 1884-7, I, pp. 286-8. Thomson cited this paper in Thomson, Papers, Op. cit. (58), p. 5.

77 Ibid.

78 Thomson to Stokes, 2 March 1854, ULC add MS 7656, K 64, Thomson's emphasis. This also shows that Thomson had already spotted Waterston's arithemetical error (see note 62) since 2000 pounds is just twice the amount that Waterston required.

79. Thomson, Papers, Op. cit. (58), pp. 10-13.

${ }^{80}$ Thomson to Stokes, 9 March 1854, ULC add MS 7656, K 66.

${ }^{81}$ Stokes to Thomson, 28 March 1854, ULC add MS 7342, S 369 . It should be pointed out that Stokes did not reply at once because he had been ill.

${ }^{82}$ W. Herschel, 'On the Nature and Construction of the Sun and Fixed Stars', Phil. Trans., 1795, $46-72$. 
an enormous body in a state of intense heat, emitting continually a portion of its original heat; as in fact 'growing dim with age' 83

This reaction is to a certain extent puzzling since Stokes seems to have adhered to the theory of primitive heat even after Thomson had shown in 1852, albeit implicitly, that the theory was untenable. Stokes and Thomson were not in contact during this period ${ }^{84}$ and I would suggest that Stokes's statement that he was 'in the habit' of holding this theory implies that he had not been particularly concerned with this problem. There is, therefore, nothing unreasonable in Stokes accepting Thomson's theory once Thomson had explained it to him. ${ }^{85}$

However, Stokes was rather more cautious than Thomson appears to have been in accepting the possibility that meteoric impacts might produce the requisite amount of solar heat. He pointed out that Thomson's version of the theory (which only altered the numbers from Waterston's version in order to take account of an arithmetical error which Waterston had made) would result in an increased mass of the central body of the solar system, and hence an observable augmentation of the earth's motion and a consequent retardation of the moon's motion, neither of which had been observed to occur. ${ }^{86}$

Thomson replied to Stokes's criticism by writing:

I think I can prove that the sun's light is due to parts of the zodiacal light (which is merely a whirling cloud of stones acc[ordin]g [to] Herschel) falling in. ${ }^{87}$

John Herschel had said that according to the laws of dynamics the zodiacal light must be composed of many solid particles rotating as individual planetlets round the sun; and by their mutual interaction, Herschel continued, some must fall onto the sun and inner planets. ${ }^{88}$ The zodiacal light was observed to be entirely within the orbit of the earth and therefore could not exercise any perturbatory influences on the motions of the earth-moon system. If it could be shown that there was enough material within the zodiacal light to keep the sun fueled with meteors, then there would be no need to posit material coming from outside the solar system. ${ }^{89}$

Thomson was also led to modify Waterston's theory because he had been, before he read the paper

trying to make out what share of meteors the earth $w[$ oul] d take, if the sun

\footnotetext{
${ }^{83}$ Stokes to Thomson, 28 March 1854, ULC add MS 7342, S 369. The quotation is a misquote of Genesis 27. 1.

${ }^{84}$ Stokes to Thomson, 24 February 1854, LLC add MS 7342, S 366.

${ }^{85}$ Thomson to Stokes, 2 and 9 March 1854, LLC add MS 7656, K 64 and 66 respectively.

${ }^{86}$ Stokes to Thomson, 28 March 1854, LLC add MS 7342, S 369.

87 Thomson to Stokes, 21 March and 29 April 1854, ULC add MS 7656, K 68. This presumably refers to Herschel, Op. cit. (11), art. 897 where he discussed the zodiacal light.

88 Ibid.

89 This Thomson argued for in Op. cit. (58) which was read to the Royal Society of Edinburgh on 17 April 1854.
} 
gets enough to produce his heat, \& I think it possibly reconcilable with what we have of falling stars, \&c. ${ }^{90}$

By the time he read his paper he had decided that if the meteors originated from outside the solar system the earth would be struck much more copiously by meteors than it was observed to be. ${ }^{91}$

Thomson pointed out that the new hypothesis, in which the meteors circled the sun as the zodiacal light, meant that the velocity of impact of the meteors on the sun would be that of a planet at a distance of the sun's radius from its centre--i.e. $1 / \sqrt{2}$ of the sun's escape velocity. Thus the amount of matter required to keep the sun going had to be doubled. Even so this would not, over the historic period, result in an observable increase of the sun's volume. ${ }^{92}$

Thomson had therefore established a theory which successfully conformed to the constraints imposed on any solar theory:

According to this form of the gravitation theory, a meteor would approach the Sun by a very gradual spiral, moving with a velocity very little more than that corresponding to a circular path at the same distance, until it begins to be much more resisted, and to be consequently rapidly deflected towards the Sun; then the phenomenon of ignition commences; after a few seconds of time all the dynamical energy the body had at the commencement of the sudden change is converted into heat and radiated off; and the mass itself settles incorporated in the Sun. ${ }^{93}$

According to this mechanism of solar heat creation meteors moved in slowly decaying orbits round the sun. This implied the existence of a resisting medium in the form of a solar atmosphere. Thomson was not very clear as to precisely what he thought the solar atmosphere was, though he certainly thought it existed..$^{94}$ Thomson at this time had also calculated the density of the aether required to transmit the sun's energy to the earth, showing that it must necessarily be greater near the sun than in the vicinity of the earth. ${ }^{95}$ This may have led him to identify the dense aether with the solar atmosphere; he did think that the aether was probably an extension of the earth's atmosphere ${ }^{96}$ and therefore this identification may not be too unreasonable. This necessity for the aether to be dense near the sun provided Thomson with his required resisting medium.

I have so far discussed how Thomson formulated his theory of solar heat, but not how he attempted to establish that this was the true theory of

\footnotetext{
${ }^{90}$ Thomson to Stokes, 9 March 1854, ULC add MS 7656, K 66.

${ }^{91}$ Thomson, Papers, Op. cit. (58) p. 7.

92 Ibid., $8 . \quad{ }^{93}$ Ibid.

94 Thomson to Stokes, 2 March 1854, ULC add MS 7656, K 64

$95 \mathrm{~W}$. Thomson, 'On the Mechanical Value of a Cubic Mile of Sunlight, and on the possible density of the Luminferous Medium', Proc. Roy. Soc. Edinb. 1854, 2, 253-5. This was later published as 'Note on the Possible Density of the Luminferous Medium and on the Mechanical Value of a Cubic Mile of Sunlight', Trans. Roy. Soc. Edinb., 1852, 21, 57-61; Papers II, 28-33.

96 Ibid., 28, and Thomson to Stokes, 21 March and 20 April 1854, ULC add MS 7656, K 68.
} 
solar heat. He had ensured that according to his theory the sun would have emitted sufficient energy at a constant rate and yet appear to have remained the same size over the historic period of astronomical observations. However, merely satisfying these constraints was not sufficient since more than one theory may do so. Thomson seems to have been aware that a well-established theory should explain and predict.

He showed that his theory provided simple explanations of phenomena which previously had had complicated explanations, or none at all. For example, he declared

The meteoric theory affords the simplest possible explanation of past changes of climate on the earth. For a time the earth may have been kept melted by the heat of meteors striking it. A period may have followed when the earth was not too hot for vegetation, but was still kept, by the heat of the meteors falling through its atmosphere, at a much higher temperature than at present. ${ }^{97}$

Other phenomena which could be accounted for included novae which might be caused by a dark body entering a cloud of meteors. ${ }^{98}$ This is particularly interesting since it implied that Thomson's view of the sun and stars was non-evolutionary at that time, i.e. there was no necessity for the stars and the meteors, which supplied them with energy, to have been created together.

More importantly he predicted phenomena which would necessarily be a consequence of his theory if it were true. His main inference was that the mass of the zodiacal light should cause perturbations in the motions of the planets. ${ }^{99}$ The zodiacal light, a flattened disc in the plane of the sun's equator, would naturally exert varying influences on the planets as they moved above and below its plane. The most extreme case of this phenomenon would be that Mercury would gradually move towards the sun, ultimately falling into its atmosphere; alternatively he considered that it might be slowly dissipated in the solar atmosphere as its orbit decayed. ${ }^{100}$ Since there were not observed to be any perturbations in the motion of the planets caused by the meteors in the zodiacal light this put an upper limit on the amount of meteors that could be present: there were, Thomson estimated, only enough to fuel the sun for a further three hundred thousand years. ${ }^{101}$

His second inference was that the differences in the density of the aether near the sun might 'produce annual apparent motions in the stars, which may be sensible though not yet discovered'. ${ }^{102}$ In other words the light of a star near the sun should be refracted by the solar atmosphere so that it should appear to have slightly changed its position in a direction away from the sun.

Thirdly Thomson attempted to determine the chemical composition

${ }^{97}$ Thomson, Papers, Op. cit. (58), p. 9.

${ }^{98}$ Ibid. $\quad{ }_{99}$ Ibid., 24.

${ }^{100}$ Ibid., 20. $\quad{ }^{101}$ Ibid., 24-5. $\quad 102$ Thomson, Proc. Roy. Soc. Edinb., Op. cit. (95), p. 254. 
of the sun using Stokes's work on line spectra. Stokes had, in 1851 or '52, devised a mechanical explanation for the identical wave-lengths of some emission and absorption lines. ${ }^{103}$ This guaranteed that the chemical substance which caused an emission line from a terrestrial flame would also be the cause of a solar absorption line of the same wave-length. Stokes had privately informed Thomson of this in a conversation when he had visited Cambridge at the time. Thomson when he was working on his mechanism for the production of solar heat in February 1854 no doubt remembered this conversation and he wrote to Stokes for further details, asking particularly if the iron spectrum was present in the solar spectrum. ${ }^{104}$ It was well known that iron was the main constituent of the meteorites which landed on earth and it was therefore reasonable to suppose that iron was the chief element in the meteors falling onto the sun. Although Stokes had assumed that a particular spectral line was caused uniquely by one type of matter, he was far from certain what this matter was. Stokes told Thomson that Léon Foucault and W. A. Miller had independently shown, for example, that the double yellow $\mathrm{D}$ lines were apparently produced by a number of different metals apart from sodium. ${ }^{105} \mathrm{He}$ was therefore not prepared to make any definite statement concerning the origin of the dark double $\mathrm{D}$ lines in the solar spectrum beyond that they were caused by the same agency which produced the double yellow lines of the same wave-lengths. In addition Stokes told Thomson that David Brewster had observed that Fraunhofer lines $\mathrm{A}$ and $\mathrm{B}$ had the same wave-length as the emission lines apparently belonging to potassium nitrate $\left(\mathrm{KNO}_{3}\right){ }^{106}$ he also thought that Brewster had identified group $a$ of the solar spectrum. ${ }^{107}$ But Stokes was not prepared to commit himself to saying that the Fraunhofer lines were indicative of these or any other chemical substances in the solar atmosphere. And he certainly could not supply Thomson with any information concerning the iron spectrum. Both Stokes and Thomson agreed that the Fraunhofer lines were potentially usable to determine the chemical constitution of the sun but they were unable to put this into practice. Thomson had attempted to use the Fraunhofer lines to confirm his theory of solar heat, rather than to investigate the chemical composition of the sun.

\footnotetext{
${ }^{103}$ For a detailed discussion of Stokes's spectral work see chapter 5 of my Ph.D. thesis, 'The Early Development of Spectroscopy and Astrophysics', University of London (Imperial College), 1981.

${ }^{104}$ Thomson to Stokes, 2 March 1854, ULC add MS 7656, K 64.

105 Stokes to Thomson, 24 February 1854 , ULC add MS 7342, S 366. L. Foucault, 'Lumière électrique', L'Institut, 1849-50, $17,44-6$. W. A. Miller, 'Experiments and observations on some cases of lines in the prismatic spectrum produced by the passage of light through coloured vapours and gases, and from certain coloured flames', Phil. Mag., 1845, I1I, 27, 81-91. For a discussion of this work sce M. A. Sutton, 'Spectroscopy and the Chemists: A Neglected Opportunity?', Ambix, 1976, 23, 16-26.

${ }^{106}$ Stokes to Thomson, 24 February 1854, LLC add MS 7342, S 366. D. Brewster, 'On the luminous lines in certain flames corresponding to the defective lines in the sun's light', Rep. Brit. Ass., 1842, part 2, p. 15.

${ }^{107}$ Stokes to Thomson, 24 February 1854, LLC add MS 7342, S 366. D. Brewster, 'Observations sur le spectra solaire', Comptes Rendus, 1850, 30, 578-81.
} 
There were two common threads to all three predictions. Firstly all were qualitative; that is Thomson did not make any attempt to suggest by how much the planets should be perturbed, nor did he suggest any value for the refraction of star light by the solar atmosphere. Secondly all these inferences were extremely difficult to verify. For Thomson, I would suggest, it was sufficient to present his work in a rigorous methodological mode, that is, as an hypothesis which conformed to the rule of greatest simplicity and from which verifiable inferences were possible.

Thomson made one final modification to his theory before it was published. He seems to have realised that if there was a solar atmosphere with meteors orbiting and falling into the sun, then there must exist friction between what he now called the 'vortex' of the meteors and the solar atmosphere; and friction creates heat and light. ${ }^{108}$ Despite this modification so that the immediate cause of solar heat was the friction between the whirling mass of the meteors round the sun and the solar atmosphere, the ultimate cause of solar heat was still contained in the dynamical energy derived from gravitational attraction existing between the sun and the meteors. This modification of the theory was published at the same time as his detailed calculations on the density of the aether. ${ }^{109}$ In this account Thomson did not repeat his suggestion that the light of the stars might be refracted round the sun. I would speculatively suggest that if the meteors and the solar atmosphere were intimately connected it would be difficult to imagine how light, especially faint star light, could be refracted through such a medium. This, if it were so, would account for the fact that Thomson made no comment on the claim made in April 1855 by the Astronomer Royal for Scotland, C. Piazzi Smyth, to have measured this refraction to be $0^{\prime \prime} .04$ at a distance of 12 minutes of time from the sun. ${ }^{110}$

Thomson's thermodynamic work acted not as an historical cause which led him to the meteoric theory, but as a context in which he rejected previously held theories of the sun, and which, when presented with Waterston's theory, he could accept and with advice modify it in accordance with the constraints imposed on any solar theory. Two of the founders of thermodynamics, Mayer and Thomson, were able using this new tool in their different ways to dispense with many mistaken ideas concerning the nature of the sun. What is interesting is that in place of these theories of solar heat, they both, independently of each other, and with different approaches, proposed remarkably similar theories, differing only in details. One of the cornerstones in the development of the principle of the conservation of energy was the determination of the mechanical equivalent

\footnotetext{
108 Appendix no. 2, 'Friction between vortices of meteoric vapour and the sun's atmosphere' in Thomson, Papers, Op. cit. (58), pp. 19-21.

${ }^{109}$ Thomson, Papers, Op. cit. (95).

110 C. P. Smyth, 'Account of experiments to ascertain the amount of Prof. Wm. Thomson's "Solar Refraction"', Proc. Roy. Soc. Edinb., 1855, 3, 302-3.
} 
of heat: a known quantity of motion generated a pre-determined amount of heat, no more and no less. I would suggest that while thermodynamics did not, indeed could not, provide a solution to the problem of solar heat, the idea that motion could be converted into heat may well have provided the initial idea that the source of solar heat was caused by moving bodies landing on the sun's surface. Neither Mayer nor Thomson despite their different approaches to the problem could tolerate the idea of the absence of a satisfactory theory of solar heat and both strove to fill the gap that they had created. Thus it seems that the constraints which the laws of thermodynamics had imposed on the sun led Mayer and Thomson to a theory which, although its shortcomings would become apparent within a very few years, satisfied their requirements at that time.

It was not long before an alternative to the meteoric hypothesis was advanced; indeed at the exact time that Thomson had been working on his theory Hermann Helmholtz, professor of Physiology at Königsberg ${ }^{111}$ had proposed a different theory of solar heat in a lecture entitled Ueber die Wechselwirkung der Naturkräfte und die darauf bezüglichen neuesten Ermittelungen der Physik. ${ }^{12}$ In this Helmholtz proposed that the sun's heat originated in the gravitational contraction of the sun. He had attended the $1853 \mathrm{Hull}$ meeting of the British Association and had sat on the committee of the physics section. ${ }^{113}$ Whether he heard Waterston deliver his paper on the maintenance of solar energy is not clear; he did not refer to Waterston in his lecture. It appears however that Helmholtz was aware of Mayer's work since he referred briefly to Mayer's work on tidal friction which Mayer had discussed in the same paper which contained his work on solar heat. ${ }^{114} \mathrm{But}$ Helmholtz made no direct reference to Mayer's meteoric theory of solar heat creation.

Like Thomson, Helmholtz was also concerned with the consequences of the second law which Thomson had drawn from it in 1852:

We must admire the sagacity of Thomson [wrote Helmholtz], who, in the letters of a long-known little mathematical formula, which speaks only of the heat, volume and pressure of bodies, was able to discern consequences which threatened the universe, though certainly after an infinite [unendlich] period of time, with eternal death. ${ }^{115}$

Here no doubt Helmholtz was referring to Thomson's 1852 paper on the dissipation of energy. ${ }^{16}$ Helmholtz drew the same conclusion as Mayer and Thomson, namely that

\footnotetext{
"II Hermann Helmholtz (1821 1894). He had not yet met Thomson, although he was to do so the following year, when they became close friends.

${ }^{112} \mathrm{H}$. Helmholtz, Ueber die Wechselwirkung der Naturkräfte und die darauf bezüglichen neuesten Ermillelungen der Physik, Königsberg 1854. Translated into English by John Tyndall as 'On the interaction of natural forces', Phil. Mag. 1856, IV, 11, 489-518.

${ }^{113}$ Athenaeum, 1853, 1097.

${ }^{114}$ Helmholtz, Phil. Mag., Op. cit. (112), p. 512 refers to Mayer's work on tidal friction which is in Mayer, Phil. Mag., Op. cit. (17), pp. 403-9.

${ }^{115}$ Helmholtz, Phil. Mag., Op. cit. (112), p. 503.116 Thomson, Op. cit. (55).
} 
the life of man, animals, and plants, could not of course continue if the sun had lost his high temperature, and with it his light. ${ }^{117}$

This realisation that the sun was the ultimate source of all energy for man while not an original sentiment, ${ }^{118}$ seems in the thought of the founders of thermodynamics to have taken on a special significance. As soon as it was realised by them that this was so, and that the sun was running down, it became important to them to discover the source of its energy.

The premises from which each man begun were different: while Thomson and Mayer started from differing thermodynamic viewpoints with which to make the leap, in their different ways, to the idea of bodies falling into the sun to cause its heat, Helmholtz, starting from the same thermodynamic viewpoint as Thomson, thermodynamically analysed the behaviour of the gaseous nebula which had been postulated by Kant and Laplace to account for the formation and evolution of the solar system. ${ }^{119}$ He pointed out that 'with regard to the origin of heat and light this view [the nebular hypothesis] gives us no information'. ${ }^{120}$ On the other hand Helmholtz believed that the nebular hypothesis was a substantially correct account of the formation of the solar system, and was indeed his only hypothesis. ${ }^{121}$ It followed that the contraction of the nebula must have affected the formation of the sun and consequently the manner in which it now sustains itself. So far as the creation of the solar system is concerned we can only posit hypotheses, such as the nebular hypothesis. But from this hypothesis deductions according to the known physical laws can be made to discover how such a nebula developed into the solar system, ending with what we now perceive, with which comparison can be made to justify the original hypothesis. One of the results of the contraction of the nebula, Helmholtz argued, was that it must account for the production of the sun's heat.

Helmholtz demonstrated that in order for the sun and planets to have coalesced as recognisable independent entities, the nebula in the process of contracting must have dissipated most of its original energy due to gravitation as heat into the universe. ${ }^{122}$ However, by setting the potential of the sun equal to the amount of energy required to raise its temperature a specified amount, he showed that there would be enough energy left within the solar system

${ }^{117}$ Helmholtz, Phil. Mag., Op. cit. (112), p. 503.

118 See for example Herschel, Op. cit. (9), art. 336.

119 Helmholtz, being German, and lecturing in Königsberg, maintained that Kant had devised a nebular hypothesis similar to Laplace's which he had devised independently. Kant had made a much more speculative suggestion than Laplace in his Allgemeine Naturgeschichte und Theorie des Himmels, Königsberg, 1755. Translated into English by W. Hastie as Universal Natural History and Theory of the Heavens, 1900, reprinted, Ann Arbor, 1969. For a discussion of the differences between the hypotheses of Kant and Laplace see G. J. Whitrow, 'The nebular hypotheses of Kant and Laplace', Actes. XIle Cong. Int. Hist. Sci., 1968, IIIB, 175-80.

${ }^{120}$ Helmholtz, Phil. Mag., Op. cit. (112), p. 505.

121 Ibid., 507. ${ }^{122}$ Ibid., 506 and 516-8. 
to raise a mass of water equal to the sun and planets taken together, not less than 28 millions of degrees of the Centrigrade scale. ${ }^{123}$

From this it followed that a contraction of $1 / 10000$ th of the radius of the sun, where most of the energy was located, would raise the temperature of a body of the same mass as that of the sun $2861^{\circ} \mathrm{C} .{ }^{124}$ Helmholtz had calculated from Pouillet's data that the sun must be cooling one and a quarter degrees Centigrade per year; ${ }^{125}$ this meant that the solar contraction which he had envisaged would account for the maintenance of solar heat for 2289 years. ${ }^{26}$ Helmholtz considered that 'such a small change ... would be difficult to detect even by the finest astronomical observations $^{\prime 127}$ and added that for at least 4000 years the sun had emitted heat without sensible change.

Helmholtz examined the behaviour of meteors to provide empirical evidence for the hypothesis that the original nebula when collapsing had dissipated heat and, that by the contraction of the sun, the nebula was effectively still emitting heat. He said that meteors - the remains of the nebula-generated a large quantity of heat when they entered the earth's atmosphere, which was then dissipated:

Thus has the falling of the meteoric stone, the minute remnant of processes which seem to have played an important part in the formation of the heavenly bodies, conducted us to the present time, where we pass from the darkness of hypothetical views to the brightness of knowledge. ${ }^{28}$

In other words, the fact that the meteors dissipated heat when brought to rest was evidence, albeit on a very much reduced scale, of the process of coalescence of the nebula which had gone on in the past. Further since meteorites were made of the same chemical materials as found on earth this must imply that the sun was made out of the same materials since all-sun, earth, meteors - had been created out of the original nebula. ${ }^{129}$

Helmholtz had thus devised a theory of solar heat which conformed to the observational constraints, and which was an integral part of the evolutionary process of the solar system. In this meteors played a purely evidential rôle and not the crucial heat supplying rôle assigned to them by Mayer and Thomson. The application of the laws of the conservation and dissipation of energy to the nebula produced, immediately, a theory of solar heat which conformed to the constraints and which did not require the formulation of any additional hypotheses. The three others who had worked on solar theories had deliberately not concerned themselves with

\footnotetext{
123 Ibid., 506.

125 Helmholtz here assumed that the sun emitted its energy uniformly over its whole mass and that its specific heat was the same as water (Ibid., 517). He seems to have used a solar radius of 717600 kilometres. He appears to have used Pouillet's $4 / 3 \mathrm{c}$ formula with $c=1$ (the specific heat of water) to obtain this figure.

126 Ibid. $\quad{ }^{127}$ Ibid., 514.

128 Ibid., $507 . \quad 129$ Ibid., 506.
} 
cosmogonical problems. Mayer said that it was impossible to provide certain evidence for cosmogonical hypotheses; Waterston had said his theory conformed to Laplace's hypothesis and Thomson regarded the nebular hypothesis as a 'mere hypothesis'. ${ }^{130}$ These three when they proceeded to devise theories of meteoric impact on the solar surface did not consider how the sun, or the meteors which supplied it, had come to exist or how the relationship which subsisted between them had been created.

Thomson's antipathetical attitude towards the nebular hypothesis stemmed from his analysis of the present motions of the bodies of the solar system. If the present planetary motions of the solar system were traced back according to the known dynamical and thermodynamical laws then, he declared, that all 'the bodies now constituting our solar system have been at infinitely greater distances from one another in space than they are now'. ${ }^{131}$ Thus

The nebular theory, as ordinarily stated, assuming as it does a previously gaseous state of matter, is not only untrue, but the reverse of the truth. ${ }^{132}$

According to Thomson therefore the solar system could not have originated from a gaseous nebula as Kant and Laplace had postulated.

That Thomson then proceeded to reject Helmholtz's theory of solar heat which was, after all, based on the nebular hypothesis, is not surprising; this he did at the Liverpool meeting of the British Association in September 1854:

It is quite certain that it cannot, as the nebular theory has led some to suppose it may, be the energy of gravitation effecting any continued condensation of the sun's present mass, since without increased pressure, it is only by cooling that any condensation can be taking place. ${ }^{133}$

Helmholtz had only been concerned with a straightforward theory in which the motion of the particles of the sun towards the solar centre caused a constant quantity of heat to be emitted by the sun without apparent lowering of temperature; he had not taken any account of the change in velocity of the particles or of the density and pressure of the sun at different densities from the centre. Thomson made no attempt to show whether, when these variables were taken into account, it would produce a theory of the sun which would account for the production of solar heat. I would suggest that Thomson thought that because his theory of meteoric impact (which he reaffirmed as true ${ }^{134}$ ) had already accounted for the production

\footnotetext{
130 Thomson to David King, 3 February 1862, in A. G. King, Kelvin the Man, London, 1925, pp. 1012

${ }^{131} \mathrm{~W}$. Thomson, 'On the mechanical antecedents of motion, heat, and light', Rep. Brit. Ass. 1854, part 2, 59 63; Papers II, 34 40, p. 38.

132 Ibid.

133 Thomson, Rep. Brit. Ass., Op. cit. (131), p. 62. This passage appeared in a footnote that Thomson omitted from his Papers.

${ }^{134}$ Thomson, Papers, Op. cit. (131), pp. 39-40.
} 
of solar heat, no other theory could be valid-especially if it depended on the nebular hypothesis which he thought untrue.

There Thomson left the problem of solar heat until $1859,{ }^{135}$ in which year U. J. J. Leverrier announced his discovery of the advance of the perihelion of Mercury, ${ }^{136}$ made in the context of his normal work on celestial mechanics. ${ }^{137}$ Leverrier remarked that an increase of $1 / 10$ th in the mass of Venus would account for the advance of Mercury's perihelion; this he rejected since it would cause all sorts of perturbations in the motion of the earth, with the theory of which he was already satisfied. The advance of the perihelion could also be due to a planet of the same size as Mercury in an orbit at half Mercury's distance from the sun; this he also had to reject since such a planet would have been observed previously. He therefore suggested that the disturbing mass could be the result of a large number of 'corpuscles' circling the sun within Mercury's orbit. Whether this suggestion was influenced by Thomson's 1854 work is not clear, though it should be pointed out that Thomson's paper on the meteoric hypothesis had been translated into French. ${ }^{138}$

Thomson said that this discovery was similar to Leverrier's and Adams's 1846 discovery of Neptune, ${ }^{139}$ in that Leverrier had discovered that Mercury was also affected by 'planetary matter' not previously recognised. ${ }^{140}$ Thomson continued by asking if this matter was 'unseen' answering himself thus:

Surely, on the contrary, it is it that we see as the Zodiacal Light, long before conjectured to consist of corpuscles circulating round the sun. ${ }^{141}$

Thomson, as can be imagined, drew the obvious conclusion by saying that Leverrier's discovery provided

that kind of evidence of the existence of matter circulating round the Sun within the earth's orbit, which, more than five years ago, in publishing his theory of meteoric vortices to account for the Sun's heat and light, he [Thomson] had called for, from perturbations to be observed in the motions of the known planets. ${ }^{142}$

Thomson's enthusiasm was to be very short lived, for a month later he published results which would ultimately refute the meteoric hypothesis.

135 Apart from ULC add MS 7342, NB 34, pp. 204-5 where he attempted to examine on 6 and 15 August, and 27 October 1855, the effect on the excentricity of the planets due to meteorites falling onto the sun. These calculations were not published.

${ }^{136}$ U. J. J. Leverrier, 'Sur la théorie de Mercure et sur le mouvement du périhélie de cette planète', Comptes Rendus, 1859, 49, 379-83.

${ }^{137}$ For a discussion of how Leverrier made his discovery see N. R. Hanson, 'Leverrier: The Zenith and Nadir of Newtonian Mechanics', Isis, 1962, 53, 359-78, especially section 2.

${ }^{138} \mathrm{~W}$. Thomson, 'Mémoire sur l'énergie mécanique du système solaire', Comptes Rendus, 1854, 39, $682 \cdot 7$.

${ }^{139}$ For an account of how Neptune was discovered see M. Grosser, The Discovery of Neptune, Boston, 1962.

$140 \mathrm{~W}$. Thomson, 'Recent investigations of M. Leverrier on the motion of Mercury', Proc. Glasg. Phil. Soc., 1859, 4, 263-66; Papers V, 134-7. Read 14 December 1859, p. 137.

141 Ibid. 142 Ibid., 134. 
He showed that according to his calculations the motion of Mercury should have displayed, over the previous one hundred and fifty years, a geocentric difference of eight and a half seconds of arc from its calculated position if enough matter was to be present to produce the heat of the sun. ${ }^{143} \mathrm{He}$ pointed out that this difference, had it existed, would have been detected by Leverrier in the course of his very precise work on Mercury's perihelion. Thomson was not, at this time, prepared to conclude that there were not enough meteors within Mercury's orbit to cause the sun's heat since he argued that

it may be concluded that if matter has been really falling in at the rate supposed by my dynamical theory of the solar radiation, the place from which it has been falling must be either nearer the Sun or more diffused from the plane of Mercury's orbit than we have supposed in the preceding example. ${ }^{144}$

Although Thomson suggested means of verifying this suggestion, it is fairly obvious that he was beginning to add supplementary hypotheses to his original theory in order to account for this discovery.

Just over two years later Thomson said that Leverrier's results indicated that the amount of matter circulating the sun was 'very small'. ${ }^{145}$ Therefore he argued

If the meteoric influx taking place at present is enough to produce any appreciable portion of the heat radiated away [from the sun], it must be supposed to be from the matter circulating round the sun, within very short distances of his surface. ${ }^{146}$

But the problem with this was that the

density of this meteoric cloud would be supposed so great that comets could scarcely have escaped as comets actually have escaped, showing no discoverable effects of resistance, after passing his surface within a distance equal to $1 / 8$ of his radius. All things considered, there seems little probability in the hypothesis that solar radiation is compensated, to any appreciable degree, by heat generated by meteors falling in. ${ }^{147}$

Thomson was thus, as in 1852, reduced to searching for an hypothesis that would account for the production of solar energy. Again there was one waiting for him; at the 1861 British Association meeting at Manchester, Thomson effectively accepted Helmholtz's theory of solar heat though with some alteration. ${ }^{148}$

$143 \mathrm{~W}$. Thomson, 'On the variation of the periodic times of the earth and inferior planets, produced by matter falling into the sun', Proc. Glasg. Phil. Soc., 1860, 4, 272-4; Papers V, 138-40. Read 4January 1860 , p. 140.

144 Ibid. $\quad 145$ Thomson, Lectures, Op. cit. (3), p. 360.

146 Ibid. 147 Ibid., 360-1.

148 W. Thomson, 'Physical Considerations regarding the Possible Age of the Sun's Heat', Rep. Brit. Ass., 1861, part 2, 27-8; Papers V, 141-4. It is worth noting that Thomson's popular Macmillan's Magazine paper (Op. cit. (3)) (which can be regarded as an expanded version of this British Association paper) was the only place, at this time, where Thomson explicitly repudiated his meteoric hypothesis. In his British Association paper it is only by accepting Helmholtz's theory that he implicitly rejects the meteoric hypothesis. The other major difference between the two papers is that in the British Association paper Thomson did not explicitly attack Darwin's estimate of the age of the earth, whereas in the Macmillan's Magazine paper he did. 
Thomson, since he had raised (in 1854) objections to Helmholtz's theory of solar contraction, had to deal with them himself before he could reasonably accept it. This was not possible in the early sixties because the mathematical description of such a mass as the sun had not yet been tackled, and Thomson apparently made no attempt to do so then. ${ }^{149}$ To deal with the problem he took limits on either side of possible specific heats and possible increases in density towards the sun's centre to arrive at the limits of the rate of contraction. Within these limits Thomson was prepared to admit Helmholtz's theory:

The meteoric theory of solar heat . . . in the form in which it was advocated by Helmholtz ... is adequate, and it is the only theory consistent with natural laws which is adequate to account for the present condition of the sun. ${ }^{150}$

Thomson effectively made Helmholtz's theory a meteoric theory, but instead of the continuing process which Thomson had originally envisaged, he placed the meteoric action far back in time when the sun had originally coalesced. Thomson made no mention of the nebular hypothesis; although he now accepted Helmholtz's theory of the maintenance of solar heat he could not bring himself to believe in the nebular hypothesis from which Helmholtz had derived it.

It is not clear at what point between 1859 and 1861 Thomson accepted Helmholtz's theory of solar heat, thus acquiring a tool with which he could attack Darwin's estimate of the age of the earth. Hitherto the age of the earth had not been a pressing problem for Thomson; in his 1854 paper he had discussed it in an appendix written in August, ${ }^{151}$ calculating from the present rate of the rotation of the sun that meteors had been falling onto the sun for 32000 years - which even so was a considerable increase over 6000 years.

In his first version of the paper in which he accepted Helmholtz's theory Thomson did not directly contradict Darwin's estimate of the age of the Weald. He contented himself by saying that:

It seems ... most probable that the sun has not illuminated the earth for $100,000,000$ years, and almost certain that he has not done so for $500,000,000$ years. ${ }^{152}$

However in his second version of his paper written for a popular audience Thomson specifically attacked Darwin: 'What then are we to think of such geological estimates as 300,000,000 years for the "denudation of the Weald"?'. ${ }^{153}$ The question naturally arises did Thomson become commit-

\footnotetext{
149 This he did later in the century with the knowledge of J. H. Lane's work. W. Thomson, 'On the equilibrium of a gas under its own gravitation only', Proc. Roy. Soc. Edinb., 1887, 14, 111-18; Papers V, 184-90.

${ }^{150}$ Thomson, Papers, Op. cit. (148), p. 143.

151 Appendix no. 4, 'On the Age of the Sun', in Thomson, Papers, Op. cit. (58), pp. 23-5.

152 Thomson, Papers, Op. cit. (148), pp. $143-4$.

153 Thomson, Lectures, Op. cit. (3), p. 368.
} 
ted to Helmholtz's hypothesis in order to attack Darwin? His 1854 meteoric hypothesis had problems which he had not been able to resolve satisfactorily, and consequently he could not very well use it to criticise Darwin's age of the Weald without opening himself to criticism. Certainly, by specifically admitting that he had been wrong in proposing the meteoric theory and by then admitting a theory he had previously rejected, he provided himself with a reputation for openmindedness and could attack Darwin with apparently objective physical reasoning.

However Thomson may or may not have modified his views on the maintenance of solar heat after 1859 in order to criticise Darwin's calculation, the origin of this work lay in his and other's development of the first two laws of thermodynamics. In Thomson's case this was motivated by his belief that God had written the laws of thermodynamics into nature in order for earth and man to fulfil the wishes of the Creator. It was Thomson's task to elucidate not only these laws but the consequences which would flow from them. As to the future he was reluctant to provide any quantitative estimate concerning the length of time for which the sun might continue to emit its energy at its present level. He thought that 'the inhabitants of the earth cannot continue to enjoy the light and heat essential to their life, for many million years longer'. ${ }^{154}$ Although he had shown that the sun would eventually cease emitting energy he had also shown that this event would occur in the distant future.

In their examination of the problem of solar heat and the hypotheses which they had proposed to account for it, Mayer, Waterston, Thomson and Helmholtz had placed the study of the sun on an entirely new theoretical basis. Indeed Helmholtz's theory of the sun was to gain such a hold on nineteenth century thought that Agnes Clerke was able to write near the end of the century that his 'theory of solar energy [is] now generally regarded as the true one'. ${ }^{155}$ It did after all fulfil all the requirements of a solar theory and while arguments were to continue all through the century and beyond about the physical constitution of the sun, ${ }^{156}$ and for how long it had supplied its energy, ${ }^{157}$ the basic problem of how such an enormous amount of energy could be generated had been solved. ${ }^{158}$

Of the four scientists who had concerned themselves with the theory of solar heat in the 1840 s and ' $50 \mathrm{~s}$, all but one-Waterston-had been

154 Ibid., 375.

${ }^{155}$ A. M. Clerke, A Popular History of Astronomy during the Nineteenth Century, 3rd edition, London, 1893, p. 378. See also J. T. Merz, A History of European Thought in the Nineteenth Century, 4 volumes, Edinburgh, 1904-1912, II, 358-9.

156 See for example, A. J. Meadows, Science and Controversy: A Biography of Sir Normam Lockyer, London, 1972 and Early Solar Physics, Oxford, 1970.

${ }^{157}$ See Burchfield, Op. cit. (4) for an account of this debate.

158 See D. H. DeVorlin, 'An Astronomical Symbiosis: Stellar Evolution and Spectral Classification (1860-1910)', Leicester University Ph.D. thesis, 1978, for an account of the development of the theory after this period. 
concerned in formulating the principles of the conservation and dissipation of energy. Mayer, Thomson and Helmholtz had all discovered as a result of their thermodynamic work that the sun was running down and until they had formulated their theories of solar heat there was no indication about how long the sun might continue to emit radiation; and in the case of Mayer this even then was not certain. In the course of their work each had realized that the sun was and had been virtually the sole supplier of energy to the earth; the basic sources of energy on earth: coal, winds, wood, oil, all ultimately emanated from the sun. ${ }^{159}$ But more than this they realized that if the sun were to cease to emit radiation then it would not be possible for life on earth to continue. Because the sun was the sole supplier of energy and was running down, it followed that man's period of habitation on earth was distinctly limited. This caused them great concern and contrasts sharply with the attitudes of those others who had taken an interest in solar theories at this time, especially Waterston and Stokes, both of whom expressed no concern whatsoever for the future of the human race. This concerned only manifested itself in the work of those who had formulated the principles of thermodynamics. Even though the reasons for concern with the problem were different for each scientist-only Thomson stated explicit theological arguments-I would suggest that these scientists who by their thermodynamic work had condemned life on the planet to a miserable end, felt it incumbent upon themselves to attempt an explanation of solar heat. This they had done and man could be assured of a considerable further period of residence on the earth.

${ }^{159}$ A point made quite explicitly by Thomson in Papers, Op. cit. (53), pp. 509-510.

In addition to what was said at footnote 61 , we note that in 1846 , Waterston, in a paper read to the Royal Society, had anticipated part of the kinetic theory of gases. In this paper he had also briefly speculated on the quantity of energy produced by the sun and on possible ways in which it might sustain itself. This was not published at this time apart from a short abstract: 'On the Physics of Media that are Composed of Free and Perfectly Elastic Molecules in a State of Motion', Proc. Roy. Soc. 1846, no. 65, p. 604. In 1891 the paper was discoverd in the archives of the Royal Society by Lord Rayleigh who had it published in full in Phil. Trans. 1892, 183, 5-77. Waterston's discussion of the sun occurs on pp. 54-5. For an account of the history of this paper see S. G. Brush, 'J. J. Waterston', D.S.B., XIV, $184-6$.

And in addition to footnore 113, note that Helmholtz abstracted Waterston's paper in Die Fortschritte der Physik im Jahre 1853, vol. 10, Berlin, 1856. It is not clear whether Helmholtz did this before or after he delivered his lecture. 


\section{Oxford University Press}

\section{A Short History of Twentieth-Century Technology}

\section{c. $1900-$ c. 1950 \\ Trevor I. Williams}

This book covers all aspects of twentieth-century technology, including thosesuch as textiles and metallurgy - which have their roots in the distant past, and others-such as aeronautics and computers-which are essentially products of this century. While the emphasis is on technological innovation, attention is also directed to the social, economic and political factors influencing recent industrial developments. Illustrated $£ 12.50$

\section{Otto Warburg}

\section{Biochemist and Eccentric}

\section{Hans Krebs, with Roswitha Schmid}

'His account of the history and significance of Warburg's scientific work, and of the atmosphere in which it was carried out, make fascinating reading for any student of contemporary biology... One does not need to be an historian of science to feel a debt of gratitude to Sir Hans Krebs for producing what is as much an eminently readable work of scholarship as it is a labour of love.' Nature. $£ 10$

\section{Gentlemen of Science}

\section{Early Years of the British Association} for the Advancement of Science

\section{Jack Morrell and Arnold Thackray}

'The British Association offers a unique medium to those who take the trouble to make full use of it. Morrell and Thackray have provided an enthralling account of how and why their "Gentlemen of Science" mastered this difficult art, and in so doing they have revealed much about the perenially interesting subject of establishing a career... Morrell and Thackray have set a challenging standard to anyone who attempts the next phase of the Association's history.' New Scientist. $£ 30$

\section{Divine and Contingent Order}

\section{Thomas F. Torrance}

'This important new book... is intended as much for scientists as for theologians and is certainly demanding reading for either... No reader who engages seriously with his argument can think in quite the same way again.' British Book News. $£ 9.50$ 